Planetary Systems in the Universe - Observation, Formation and Evolution

Proceedings IAU Symposium No. 202, (C)2004 IAU

Alan Penny, Pawel Artymowicz, Anne-Marie Lagrange, \& Sara Russell, eds.

\title{
The 3.3 micron PAH feature in Vega-type stars
}

\author{
Dolores M. Walther \\ Gemini Observatory, 670 North A'ohoku Pl. University Park, Hilo, \\ Hawaii, 96720, USA \\ Iain M. Coulson, Jane Greaves, Wayne Holland \\ Joint Astronomy Centre, 660 North A'ohoku Pl. University Park, Hilo, \\ Hawaii, 96720, USA \\ William R.F. Dent \\ Royal Observatory Edinburgh, Edinburgh EH9 3HJ, UK.
}

\begin{abstract}
We present spectroscopic data of 3 isolated Vega-type systems which exhibit the $3.3 \mu \mathrm{m}$ 'PAH' feature ascribed to stretching of the C-H bonds in aromatic hydrocarbons. These are part of a sample of mainly hot stars. We discuss the implications of Vega-type stars having an organic component to their surroundings and compare them with the unique star, HD135344 (SAO 206462).
\end{abstract}

\section{Introduction}

The well-known unidentified infrared (UIR) emission band at $3.3 \mu \mathrm{m}$ is generally attributed to the $\mathrm{C}-\mathrm{H}$ stretching mode of aromatic hydrocarbons (Duley \& Williams 1981). They can either be in the form of free molecules, especially polycyclic aromatic hydrocarbons (PAHs) (Leger \& Puget 1984; Allamandola et al. 1985), or in small dust grains (Sakata et al. 1984, 1987; Borghesi et al. 1987; Papoular et al. 1989). The existence of these very small grains and large molecules was deduced by Sellgren (1984) who observed emission from reflection nebulae at $3.3 \mu \mathrm{m}$ at such large distances from the exciting star that the equilibrium temperature would be too low for the emission to be produced by dust grains in thermal equilibrium. The fluorescence of PAHs following the absorption of UV photons has explained many of the widely observed and previously UIR emission features in planetary and reflection nebulae (Geballe, 1996) as well as the detection of PAH emission in the Vega-type star HD 135344 (Coulson \& Walther, 1995). Vega-type stars are main-sequence stars with unexpected emission in the far-IR and/or in the near-IR. Approximately $50 \%$ of Vega-type stars are of spectral types A and B (Mannings \& Barlow, 1998). The 3 B-stars we present have IR excess typical of Vega-type stars. 


\section{Observations and Results}

The 3.3 micron spectra were obtained at the $3.8 \mathrm{~m}$ United Kingdom Infrared Telescope on Mauna Kea, Hawaii, using a cooled grating infrared spectrometer, CGS4, on 2000 August 13 UT. The short focal length camera was employed $(\mathrm{F}=150 \mathrm{~mm})$ with the $45 \mathrm{l} / \mathrm{mm}$ grating and a $256 \times 256 \mathrm{InSb}$ array to give a resolving power of 600 .

Wavelength calibration was achieved by comparison with an argon lamp and is considered accurate to $0.001 \mu \mathrm{m}$. The spectra were divided by an appropriate spectrum of a nearby standard star to remove telluric lines and make air mass corrections and then multiplied by a Planck function appropriate to the temperature of the standard.

The spectral range we observed includes the atomic hydrogen line $\mathrm{Pf} \epsilon$ at $3.04 \mu \mathrm{m}$, a blend of Pf $\delta(3.29 \mu \mathrm{m})$ with a PAH line at $3.3 \mu \mathrm{m}$ and a PAH line at $3.4 \mu \mathrm{m}$. The Pf $\epsilon$ line profile was used to subtract the hydrogen contribution to the blend. No emission was detected at $3.4 \mu \mathrm{m}$ in these stars. Total integration time was 10 minutes per source. The integrated intensities of the features are displayed in Table 1.

\section{Discussion}

Like HD 135344, HD 232344 and HD 239729 have measurable emissions at $3.3 \mu \mathrm{m}$ but not at $3.4 \mu \mathrm{m}$, a combination that implies that the PAH carriers are not free molecules but are associated with dust grains (Geballe et al. 1994). Both these stars have near-infrared excess probably due to the absorption of ultraviolet photons by these dust grains (Sellgren, 1984).

The emission line strengths from these two B-type stars are comparable to those measured in HD 135344. The latter is type F8V and finding PAHs in its environment was unexpected. PAHs have previously been detected in hot stars such as Ae/Be stars (Brooke et al., 1993), but have not been detected in their F,G,K-type counterparts, the T Tauri stars (Nata and Krugel, 1995). This leaves us with the following questions :

- Are Vega-type stars of spectral type B related to Be stars? These two B-type stars certainly exhibit Ae/Be characteristics : cold dust, hydrogen emission and PAH emission.

- Are Vega-type stars of spectral types F, G, K related to T Tauri stars? Both types have cold dust and hydrogen emission, but PAH emission has never been detected in T Tauri stars (Nata and Krugel, 1995). If there is an evolutionary link, the PAH emission from HD 135344 is anomalous.

The combination of PAHs, Pfund lines and infrared excess make HD 232344 and HD 239729 as interesting as HD 135344, and increases the sample of Vega-type stars with dusty organic environments. HD 239729 lacks a cold dusty disk, but has both IR excess and PAH emission. After further investigation we found that this star is a double and does not belong in the our sample. But it does confirm that PAH molecules continue to be found among small dust grains.

On the other hand, HD 199021 has a cold dusty disk and is the only one in this group which does not show any signs of PAHs. However, it exhibits hydrogen 
recombination lines suggesting an active chromosphere and could therefore also be classified as a Be star.

\section{Conclusion}

Two of the 3 stars studied here have the $3.3 \mu \mathrm{m}$ PAH feature seen in Be stars. All three exhibit hydrogen emissions seen in $\mathrm{Ae} / \mathrm{Be}$ and $\mathrm{T}$ Tauri stars. Vega-type stars have typically been classified by their infrared excess due to continuum emission from dust, we are finding that many of them have features in common with the AeBe and TTauri stars : near-infrared excess, atomic hydrogen emission lines and, now, PAH emission lines. Understanding these relationships will help place these Vega-type stars in their evolutionary context.

Table 1. Line Intensities $\left(\mathrm{W} / \mathrm{m}^{2} \mu \mathrm{m}\right)$

\begin{tabular}{ccccc}
\hline \hline Target & Sp. & $\begin{array}{c}\text { Pfund 10-5 } \\
3.0392 \mu \mathrm{m}\end{array}$ & $\begin{array}{c}\text { Pfund 9-5 } \\
3.2970 \mu \mathrm{m}\end{array}$ & $\begin{array}{c}\text { PAH } \\
3.29 \mu \mathrm{m}\end{array}$ \\
& & & & \\
HD 232344 & B5 & $1.5 \times 10^{-16}$ & $6.4 \times 10^{-17}$ & $9.0 \times 10^{-17}$ \\
HD 199021 & B0V & $1.3 \times 10^{-16}$ & $1.3 \times 10^{-16}$ & $<1.2 \times 10^{-15}$ \\
HD 239729 & B0V & $9.0 \times 10^{-16}$ & $6.8 \times 10^{-17}$ & $1.5 \times 10^{-17}$ \\
\hline
\end{tabular}

\section{References}

Allamandola, L.J. Tielens, A.G.G.M., \& Barker, J.R. 1985, ApJ, 290, L25

Bellamy, L.J. 1958, in The Infrared Spectra of complex Organic Molecules (2nd ed.; New York; Wiley)

Borghesi, A., Bussoletti, E., \& Colangeli, L. 1987 ApJ, 314, 422

Brook, T,Y., Tokunaga, A.T., \& Strom, S.E. 1993, AJ, 106, 656

Coulson, I.M. \& Walther, D.M. 1995, MNRAS, 274, 977

Duley, W.W., \& Williams, D.A. 1981, MNRAS, 196, 269

Hartmann, L., Calvet, N., Avrett, E., H., \& Loeser, R. 1990, ApJ, 349, 168

Herzberg, G. 1966, in Electronic Spectra and Electronic Structure of Polyatomic Molecules (NY: van Nostrand Reinhold)

Geballe, T.R., Joblin, L.B., D'Hendecourt, M., Jourdain de Muizon, Tielens, A.G.G.M., \& Leger, A. 1994, ApJ, 434, L15

Geballe, T .R. 1997, in From Stardust to Planetesimals, Editors Y.J. Pendleton and A.G.G.M. Tielens

Leger, A., \& Puget, J.L. 1984, A\&A, 137, L5

Mannings, V., \& Barlow, M.J. 1998, ApJ, 497, 330

Papoular, R., Conard, J., Guiliano, M., Kister, J., \& Mille, G. 1989, A\&A, 217, 204

Sakata, A., Wada, S., Tanabe, T., \& Onaka, T. 1984, ApJ, 287, L51.

Sellgren, K. 1984, ApJ, 277, 623 